\title{
Phaeochromocytoma presenting with pseudo-intestinal obstruction and lactic acidosis
}

\author{
Peng Chin $\underline{K e k}^{1}$, MBBS, MRCP, Emily Tse Lin $\underline{H o}^{1}$, MBBS, MRCP, Lih Ming Loh $\underline{L}^{1}$, MMed, FRCP
}

\begin{abstract}
Phaeochromocytomas are rare neuroendocrine tumours with variable clinical signs and symptoms. Hypertension, tachycardia, sweating and headaches are cardinal manifestations. Although nausea and abdominal pain are the more common gastrointestinal features, rare gastrointestinal spectrums have been reported that can mimic abdominal emergencies. Metabolic effects of hypercatecholaminaemia are vast and one such rare presentation is lactic acidosis. We describe a case of phaeochromocytoma presenting with both intestinal pseudo-obstruction as well as lactic acidosis. This case report highlights the importance of having a high index of suspicion for and early recognition of the gastrointestinal and metabolic manifestations of phaeochromocytomas.
\end{abstract}

Keywords: intestinal pseudo-obstruction, lactic acidosis, phaeochromocytoma

\section{INTRODUCTION}

Phaeochromocytomas are rare neuroendocrine tumours with a prevalence rate of $0.1 \%-0.2 \%$ in subjects with hypertension. (1) They have highly variable clinical signs and symptoms, the most common being hypertension, tachycardia, sweating and headache. (1) Nausea has been suggested to be the most common gastrointestinal symptom, ${ }^{(1,2)}$ while Bravo et al reported that $14 \%$ of patients presented with abdominal pain..$^{(2)}$ In an earlier report, chronic constipation was noted in $13 \%$ of patients with phaeochromocytoma. ${ }^{(3)}$ There is, however, a spectrum of other gastrointestinal manifestations ranging from transient intestinal motility abnormalities to ischaemic ileus, resistant constipation and even bowel infarction. ${ }^{(4)}$ Fortunately, these gastrointestinal manifestations rarely develop. Metabolic manifestations such as lactic acidosis have also rarely been described. A literature review revealed 32 publications for intestinal pseudo-obstruction but only nine publications related to lactic acidosis. We herein report a case of phaeochromocytoma presenting with subacute intestinal pseudo-obstruction and lactic acidosis.

\section{CASE REPORT}

A 62-year-old Malay woman was admitted to the general surgical unit with complaints of generalised abdominal pain, constipation and vomiting over a period of five days. The patient had a medical history of Type 2 diabetes mellitus, hypertension and dyslipidaemia. She had been treated with glipizide for her diabetes mellitus, with metformin added one month prior to admission for poor glycaemic control. She had complications of diabetic retinopathy, neuropathy and nephropathy with the presence of proteinuria. The patient's anti-hypertensive medications included atenolol, lisinopril and nifedipine (adalat LA). Her baseline creatinine level was normal at 83 (normal range [NR] 40-85) $\mu \mathrm{mol} / \mathrm{L}$. On initial examination, she had a blood pressure of 196/132 $\mathrm{mmHg}$, heart rate of $142 \mathrm{bpm}$ in sinus rhythm and respiratory rate of 24 /minute. She was febrile with a temperature of $38.5^{\circ} \mathrm{C}$ and was clinically dehydrated. Her

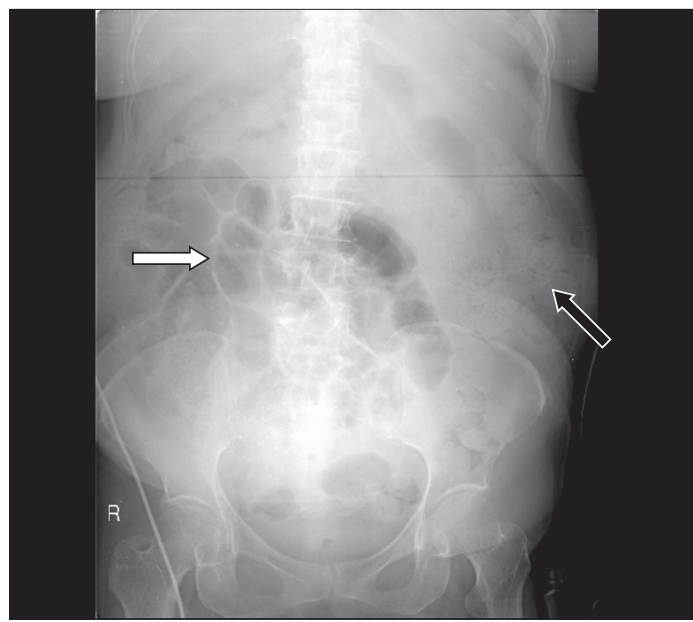

Fig. 1 Abdominal radiograph on admission shows distended small bowel loops (white arrow) with moderate faecal loading (black arrow).

cardiovascular and respiratory examinations were unremarkable. Abdominal examination revealed a distended but soft abdomen associated with poorly localised tenderness and sluggish bowel sounds. The initial working diagnoses by the surgeon included intestinal obstruction and pancreatitis.

Abdominal radiography (Fig. 1) showed distended small bowel loops with moderate faecal loading. The laboratory investigation results were as follows: total white blood cell count 19.38 $\left(N R\right.$ 4-10) $\times 10^{9} / \mathrm{L}$, haemoglobin level 17.7 (NR 12-16) g/dL, platelet count $579(\mathrm{NR} 140-440) \times 10^{9} / \mathrm{L}$ and raised neutrophil count at $88 \%$ (NR 40-75). The patient's urea level was 9.1 (NR 2.8-7.7) $\mathrm{mmol} / \mathrm{L}$, sodium 137 (NR 135-145) mmol/L, potassium 4.7 (NR 3.3-4.9) mmol/L, creatinine level $150 \mu \mathrm{mol} / \mathrm{L}$, bicarbonate level 21.4 (NR 19-31) mmol/L and amylase level 349 (NR 44161) U/L. Her lipase levels were normal at 254 (NR 23-300) U/L, as were her transaminase levels. The patient was kept nil by mouth, and given intravenous fluids and antibiotics. Metoclopramide was not administered either prior to or during admission.

The patient's condition deteriorated over the following 24 hours. She became hypotensive with a blood pressure drop 
to $90 / 60 \mathrm{mmHg}$ despite aggressive hydration and remained tachycardic. Her serum creatinine kinase level rose from $119 \mathrm{U} / \mathrm{L}$ (38-164) to a peak of $474 \mathrm{U} / \mathrm{L}$ and serum troponin-T was also raised at $0.23 \mu \mathrm{g} / \mathrm{L}(<0.03)$. Electrocardiography revealed a partial right bundle branch block but no dynamic changes. The patient's plasma bicarbonate level decreased to $13.0 \mathrm{mmol} / \mathrm{L}$, with a $\mathrm{pH}$ of 7.386 upon blood gas analysis. She had high anion gap metabolic acidosis with a calculated anion gap of 22. The patient became hypoxic with $\mathrm{pO}_{2}$ decreasing from $89.3 \mathrm{mmHG}$ to $55.1 \mathrm{mmHg}$ and required a higher concentration of supplemental oxygen. Chest radiography showed mild infiltrates in the right lung field. The cardiologist's impression was that of a non-ST-elevation myocardial infarction. However, an echocardiography done the following day showed that her left ventricular ejection fraction was good at $75 \%$. There were no regional wall motion abnormalities. In view of the discrepancies between the hypotension, hypoxia and clinical signs, a ventilation-perfusion scan was performed that ruled out the diagnosis of pulmonary embolism.

The patient's glycaemic control worsened and she was referred to an endocrinologist for poorly controlled diabetes and worsening acidosis. Her serum ketones level was normal at $0.2(0.0-0.6) \mathrm{mmol} / \mathrm{L}$, ruling out the diagnosis of diabetic ketoacidosis. Serum lactate level was, however, high at $7.1(0.7-2.1) \mathrm{mmol} / \mathrm{L}$. During the review, she was noted to have wide fluctuations of systolic blood pressure from 120-180 mmHg with persistent tachycardia. The possibility of phaeochromocytoma was entertained.

As the patient's condition was deteriorating, urgent computed tomography (CT) of the abdomen (Fig. 2) was done to rule out intra-abdominal sepsis. It showed a large left adrenal tumour that was partially cystic and measured $5.4 \mathrm{~cm} \times 3.9 \mathrm{~cm} \times 4.5 \mathrm{~cm}$. There was mild dilatation of the small bowel, which was in keeping with subacute intestinal obstruction, but no suggestion of pancreatitis. She was treated conservatively with fluids and antibiotics. Blood cultures subsequently returned negative. In view of the patient's hypotension, her anti-hypertensives were stopped. Her blood pressure remained normal for the rest of her admission. She responded well to the treatment and was discharged after a week.

Subsequent 24-hour urine collection, to test for catecholamines, detected raised metanephrine levels of 6,061 (NR 400-1,500) nmol/day and normetanephrine levels of $2,884(600-1,900) \mathrm{nmol} /$ day, confirming the diagnosis of a phaeochromocytoma. The patient's epinephrine level of 107 (3-109) nmol/day and norepinephrine level of 260 (89-473) $\mathrm{nmol} /$ day were normal. Her measured creatinine clearance was $87 \mathrm{~mL} /$ minute. Alpha $(\alpha)$-blockade with phenoxybenzamine was administered, supplemented with nifedipine to control her blood pressure. Cardiac evaluation via a myocardial perfusion scan was suggestive of mild ischaemia in the distal lateral wall. She subsequently underwent uneventful surgical excision of the adrenal tumour. The diagnosis of phaeochromocytoma was confirmed on histology. Postoperative urine catecholamines were normal and there was no recurrence of intestinal obstruction.

A review of the patient's case notes revealed three prior admissions over a three-year period. She had been admitted for

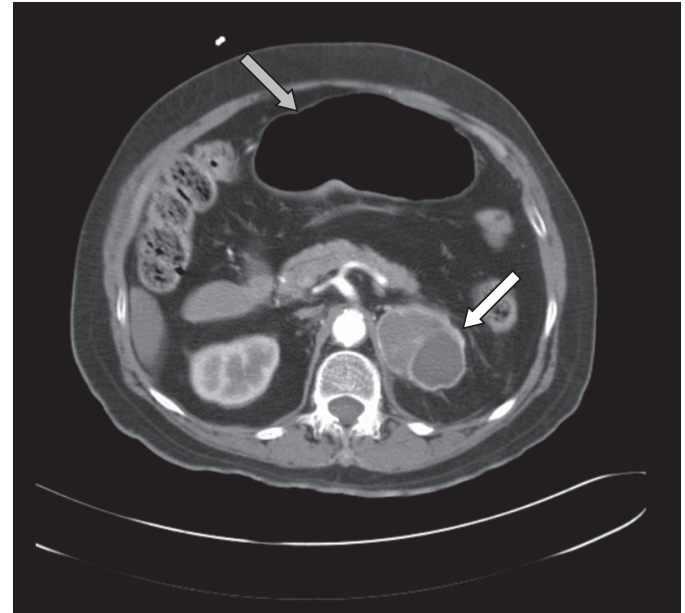

Fig. 2 CT of the abdomen showing a large cystic adrenal tumour (white arrow) and distended small bowel loops (grey arrow) consistent with subacute intestinal obstruction.

complaints of headache and giddiness on the first two occasions. Fluctuations in her systolic blood pressure between $110 \mathrm{mmHg}$ and $170 \mathrm{mmHg}$ were noted during the admissions. On the third occasion, she presented with vomiting, watery stools, fever and abdominal pain; her clinical progression was very similar to the current admission, with similar abdominal radiographic changes, progressive hypoxia and metabolic acidosis. However, her creatinine level during that admission was normal.

\section{DISCUSSION}

Phaeochromocytoma is a potentially life-threatening condition. Although the classic clinical presentation involves hypertension, tachycardia, headache and sweating, ${ }^{(1)}$ its clinical features can be diverse and highly variable. Differences in the secretion of epinephrine and norepinephrine by tumours and the differing potencies of the catecholamines on the $\alpha$ - and beta $(\beta)$ adrenergic receptors contribute to these highly variable clinical manifestations. ${ }^{(5)}$ Such variability can make the diagnosis difficult and result in a delay between the initial symptoms and final diagnosis, despite improvements in diagnostic techniques. The issue was demonstrated in this case report, where the initial presentation of headache with fluctuating blood pressure did not sound the alarm. Hence, the less common manifestations such as intestinal obstruction and lactic acidosis, which the patient also presented with, should not be overlooked.

Phaeochromocytoma may clinically manifest as a spectrum of gastrointestinal symptoms ranging from transient intestinal motility abnormalities and resistant constipation to infarction of the bowel. The effect of catecholamines on smooth muscle, both intestinal and vascular, may account for these intestinal manifestations. The net effect of sympathetic overactivity on the $\alpha$ - and $\beta$-adrenergic receptors is a depressed peristaltic state and constriction of the sphincter, leading to ileus and constipation, as seen in this case. ${ }^{(3,4,6)}$ There may be a decrease in splanchnic blood flow secondary to vasoconstriction. Sustained vascular smooth muscle contraction or spasm may also result in mucosal ischaemia with haemorrhage, ulceration and perforation, ${ }^{(4)}$ although it did not occur in this patient. 
It has also been reported that phaeochromocytomas may secrete other hormones such as neuropeptide A and endothelin-1.(7) The roles of these hormones are unclear but they may play a part in the pathogenesis of the gastrointestinal symptoms. Ileus occurs as a result of a large tumour burden with excessive amounts of catecholamine release over a prolonged period of time. Intermittent release of catecholamines can result in paroxysmal inhibition of the intestinal smooth muscles, causing intermittent symptoms, as seen in this patient. ${ }^{(4)}$

Another interesting feature observed in this patient was the presence of lactic acidosis. There has been considerable argument over the definition of lactic acidosis. A commonly used definition is a lactate level of $>5 \mathrm{mmol} / \mathrm{L}$ and $\mathrm{pH}$ level of $<7.35$. $^{(8)}$ Those opposed to it included Stacpoole, who reported that not all patients with hyperlactatemia presented with acidosis. He argued that hyperlactatemia is not synonymous with lactic acidosis, which may be associated with acidemia, a normal $\mathrm{pH}$ or alkalemia. ${ }^{(8)}$ Lactic acidosis occurring with phaeochromocytoma is very rare and has only been noted in a small number of case reports. ${ }^{(9-11)}$ In this patient, $\mathrm{pH}$ level did not fall below 7.35 , but the presence of a high lactate level, high anion gap and low bicarbonate level was definitely suggestive of lactic acidosis. However, its exact mechanism is not clear. It has been proposed that lactic acidosis is a direct consequence of excessive catecholamines. ${ }^{(9,10)}$ Hypoxia may also be a contributing cause, as was true in this case. Catecholamines have been known to stimulate glycogenolysis and increase the concentration of fuel substrates like lactate, contributing to gluconeogenesis. ${ }^{(9)}$ This may be secondary to their activation of the Cori cycle, in particular by epinephrine. Catecholamine-induced insulin suppression and glucagon stimulation further enhance these processes, and may account for poor glucose control. Catecholamines may also create an anaerobic environment through peripheral vasoconstriction, leading to an increase in lactate production. ${ }^{(9)}$ Thus, the excess lactate results from a decrease in oxygen delivery and an increase in oxygen consumption.

The other question that can be raised is whether metformin, instead of phaeochromocytoma, was the cause of the lactic acidosis. Occurrence of lactic acidosis due to metformin is not common, with an incidence of four to nine cases per 100,000 patients a year. A recent Cochrane review suggested that there is no evidence to support the association of metformin with lactic acidosis. ${ }^{(12)}$ Misbin et al found that the majority (91\%) of patients in their study who presented with lactic acidosis had one or more risk factors such as congestive cardiac failure, hypoxia or acute renal failure. ${ }^{(13)}$ Our patient was similarly at risk, with risk factors such as mild acute renal impairment and hypoxia.

Lalau et al described a case of a diabetic patient on metformin who presented with mild renal impairment and intestinal obstruction, and was noted to have lactic acidosis. ${ }^{(14)}$ They suggested that the discrepant accumulation of lactate in the presence of mild renal impairment could be related to intestinal handling of metformin during ileus. It could also be related to an interaction between organic cation transport systems of the renal proximal tubule that normally eliminate biguanides and an unknown substance related to intestinal occlusion. ${ }^{(14)}$ The clinical presentation in Lalau et al's case report, like that of this patient, was of both mild renal impairment and intestinal obstruction. The exact cause of our patient's acidosis was difficult to determine, but the excessive catecholamines, either directly or indirectly, played a part in causing the lactic acidosis. It is unknown what triggered her acute presentation and subsequent deterioration. Unopposed $\beta$-blockade, used in the management of her hypertension, may have exacerbated her symptoms. This could explain why her blood pressure was better controlled after atenolol was stopped. Another well-reported trigger for hyperadrenergic crisis is metocloproamide, ${ }^{(15)}$ but it was not the cause in this patient.

This case report illustrates one of the diverse manifestations of phaeochromocytomas. The fluctuations of blood pressure and headaches during the first two admissions should have alerted the attending physician to the possibility of a phaeochromocytoma. That could have prevented the subsequent development of the intestinal pseudo-obstruction. Secondly, for patients presenting with unexplained pseudoobstructions or unexplained lactic acidosis, the diagnosis of a phaeochromocytoma should be considered. This case report highlights the importance of having a high index of suspicion for and early recognition of the gastrointestinal and metabolic manifestations of phaeochromocytomas, as a delayed diagnosis can have disastrous consequences.

\section{REFERENCES}

1. Lenders JW, Eisenhofer G, Mannelli M, Pacak K. Phaeochromocytoma. Lancet 2005; 366:665-75.

2. Gifford RW Jr, Manger WM, Bravo EL. Pheochromocytoma. Endocrinol Metab Clin North Am 1994; 23:387-404

3. Mullen JP, Cartwright RC, Tisherman SE, Misage JR, Shapiro AP. Pathogenesis and pharmacologic management of pseudo-obstruction of the bowel in pheochromocytoma. Am J Med Sci 1985; 290:155-8.

4. Turner CE. Gastrointestinal pseudo-obstruction due to pheochromocytoma. Am J Gastroenterol 1983; 78:214-7.

5. Eisenhofer G, Rivers G, Rosas AL, et al. Adverse drug reactions in patients with phaeochromocytoma: incidence, prevention and management. Drug Saf 2007; 30:1031-62

6. Khafagi FA, Lloyd HM, Gough IR. Intestinal pseudo-obstruction in pheochromocytoma. Aust N Z J Med 1987; 17:246-8.

7. Salazar A, Naik A, Rolston DD. Intestinal pseudoobstruction as a presenting feature of a pheochromocytoma. J Clin Gastroenterol 2001; 33:253-4.

8. Stacpoole PW. Lactic acidosis. Endocrinol Metab Clin North Am 1993; 22:221-45.

9. Bornemann M, Hill SC, Kidd GS 2nd. Lactic acidosis in pheochromocytoma. Ann Intern Med 1986; 105:880-2.

10. Keller U, Mall T, Walter M, et al. Phaeochromocytoma with lactic acidosis. Br Med J 1978; 2:606-7.

11. Madias NE, Goorno WE, Herson S. Severe lactic acidosis as a presenting feature of pheochromocytoma. Am J Kidney Dis 1987; 10:250-3.

12. Salpeter SR, Greyber E, Pasternak GA, Salpeter EE. Risk of fatal and nonfatal lactic acidosis with metformin use in type 2 diabetes mellitus. Cochrane Database Syst Rev 2010: CD002967.

13. Misbin RI, Green L, Stadel BV, et al. Lactic acidosis in patients with diabetes treated with metformin. N Engl J Med 1998; 338:265-6

14. Lalau JD, Race JM, Brinquin L. Lactic acidosis in metformin therapy. Relationship between plasma metformin concentration and renal function. Diabetes Care 1998; 21:1366-7.

15. Lenders JW, Duh QY, Eisenhofer G, et al. Pheochromocytoma and paraganglioma: an endocrine society clinical practice guideline. J Clin Endocrinol Metab 2014; 99:1915-42. 